\title{
Regulation of toll-like receptors-mediated inflammation by immunobiotics in bovine intestinal epitheliocytes: role of signaling pathways and negative regulators
}

\author{
Julio Villena ${ }^{1,2}$, Hisashi Aso ${ }^{3}$ and Haruki Kitazawa ${ }^{4}$ \\ 1 Immunobiotics Research Group, Tucuman, Argentina \\ ${ }^{2}$ Laboratory of Immunobiotechnology, Reference Centre for Lactobacilli (CERELA-CONICET), Tucuman, Argentina \\ ${ }^{3}$ Cell Biology Laboratory, Graduate School of Agricultural Science, Tohoku University, Sendai, Japan \\ ${ }^{4}$ Food and Feed Immunology Group, Laboratory of Animal Products Chemistry, Graduate School of Agricultural Science, Tohoku University, Sendai, Japan
}

\section{Edited by:}

Kieran G. Meade, Teagasc - The

Agriculture and Food Development

Authority, Ireland

Reviewed by:

Dirk Werling, Royal Veterinary

College, UK

Takao Mukai, Kitasato University,

Japan

*Correspondence:

Haruki Kitazawa, Food and Feed

Immunology Group, Laboratory of Animal Products Chemistry,

Department of Science of Food

Function and Health, Division of

Bioscience and Biotechnology for

Future Bioindustries, Graduate School

of Agricultural Science, Tohoku

University, 1-1 Tsutsumidori-

Amamiyamachi, Aobaku, Sendai

981-8555, Japan

e-mail: haruki@bios.tohoku.ac.jp
Intestinal epithelial cells (IECs) detect bacterial and viral associated molecular patterns via germline-encoded pattern-recognition receptors (PRRs) and are responsible for maintaining immune tolerance to the communities of resident commensal bacteria while being also capable to mount immune responses against pathogens. Toll-like receptors (TLRs) are a major class of PRRs expressed on IECs and immune cells, which are involved in the induction of both tolerance and inflammation. In the last decade, experimental and clinical evidence was generated to support the application of probiotics with immunoregulatory capacities (immunobiotics) for the prevention and treatment of several gastrointestinal inflammatory disorders in which TLRs exert a significant role. The majority of these studies were performed in mouse and human cell lines, and despite the growing interest in the bovine immune system due to the economic importance of cattle as livestock, only few studies have been conducted on cattle. In this regard, our group has established a bovine intestinal epithelial (BIE) cell line originally derived from fetal bovine intestinal epitheliocytes and used this cell line to evaluate the impact of immunobiotics in TLR-mediated inflammation. This review aims to summarize the current knowledge of the beneficial effects of immunobiotics in the regulation of intestinal inflammation/infection in cattle. Especially, we discuss the role of TLRs and their negative regulators in both the inflammatory response and the beneficial effects of immunobiotics in bovine IECs. This review article emphasizes the cellular and molecular interactions of immunobiotics with BIE cells through TLRs and gives the scientific basis for the development of immunomodulatory feed for bovine healthy development.

Keywords: immunobiotics, TLR4, intestinal immunity, inflammation, bovine intestinal epitheliocytes, TLR negative regulators, lactobacilli, bifidobacteria

\section{INTRODUCTION}

Intestinal epithelial cells (IECs) are structurally and functionally polarized. These cells have an apical surface facing the intestinal lumen and a basolateral surface facing the underlying basement membrane and the lamina propria. IECs provide a physical barrier that separates commensal bacteria in the lumen from the underlying lamina propria and deeper intestinal layers (1). In addition, IECs are a central component of the immune system of the gut. Over the last decades, great progress has been achieved in understanding IECs immunobiology (2). It was amply demonstrated that the cross-talk between the epithelium with gut microbes significantly influences the activities of immune cells in the mucosa (2). The detection of commensal bacteria, pathogens, or probiotics by IECs is achieved through the families of germlineencoded pattern-recognition receptors (PRRs) that recognize conserved molecular structures known as microbe-associated molecular patterns (MAMPs). MAMPs and PRRs interaction and the subsequent signaling in IECs is involved in several important mechanisms that are crucial for maintaining a healthy epithelial barrier including maintenance of tight junctions strength, epithelial cell proliferation and renewal, expression of antimicrobial peptides, and modulation of mucosal immune responses (3).

In recent years, worldwide interest has rapidly and significantly increased in the therapeutic and preventive effects of "friendly bacteria." These microorganisms, recognized as probiotics, are generally selected from Lactobacilli or Bifidobacteria strains (4). Several studies in animal models as well as clinical trials support a unique role for probiotics by beneficially modulating the mucosal immune system. Thus, a new term was required to identify probiotic bacteria that promote health by regulating the mucosal immune system. Clancy suggested the new term "immunobiotics" as appropriate for fulfilling this need (5). The quest for a better understanding of how immunobiotics works have led to an enormous interest in the molecular processes underlying hostmicrobe interactions. As reviewed by Lebeer et al. (6), the final conclusion of works that have studied the molecular mechanism 
of probiotic immunomodulatory activities is that: "their effect depends on the combination of distinct MAMPs that interact with various $P R R s$ and the associated co-receptors that fine tune signaling, as well as on the quantity and quality of these MAMPs. Therefore, host-immunobiotic interactions are not univocal but involve complex interactions among various microbial molecules, host receptors, and adaptor molecules" (6).

This expanding knowledge about the cellular and molecular effects of beneficial bacteria in innate mucosal immune system has raised the possibility of new treatments for improving health not only in humans but also in animals. In this review, we describe the recent advances in the impact of immunobiotics on bovine intestinal epithelial (BIE) cells and possible novel therapeutic approaches to beneficially modulate bovine epithelial cell immunobiology. Especially, we discuss the role of toll-like receptors (TLRs), their signaling pathways, and their negative regulators in both the inflammatory-intestinal injury and the beneficial effects of immunobiotics. This article emphasizes the cellular and molecular interactions of immunobiotics with bovine epithelial cells through TLRs and gives the scientific basis for the future development of immunomodulatory feed for improving bovine health.

\section{BOVINE INTESTINAL EPITHELIAL CELLS}

The development of bovine intestinal cell cultures and their characterization with regard to their permissiveness for bacterial adhesion and invasion, and the ability to sense PAMPs through PRRs represents an important step forward toward the establishment of in vitro systems to study molecular interactions of pathogenic, commensal, and probiotic microorganisms with the bovine host (7).

For cattle, primary cultures of ileum or colon epithelial cells have been used for toxicological assays, the study of microbial virulence factors, the efficacy of antimicrobial compounds, and the evaluation of innate immune responses through PRRs signaling (7-12). A combination of the enzymatic digestion (dispase and collagenase) together with soft mechanical agitation proved to be a successful method for releasing intact, viable bovine colonic crypts from underlying mesenchymal tissue. However, a series of purification steps was required to eliminate the majority of contaminating non-epithelial cells (mostly fibroblasts) from the crypt suspension (10). Similarly, cultures of bovine colonocytes and jejunocytes were obtained by Rusu et al. (11), using a combination of enzymatic and mechanical disruption of the intestinal epithelium. The study showed that primary cultures of bovine enterocytes isolated from colon and jejunum presented characteristics of epithelial cells, such as a typical pavement-like aspect, the formation of domes and apical tight junctions, and microvilli in confluent cultures. Moreover, these bovine colonocytes and jejunocytes expressed epithelial cell markers such as brush border enzymes and the epithelium typical cytoskeleton proteins, such as cytokeratins.

Dibb-Fuller et al. (8) developed primary bovine cell lines from ileum, colon, and rectum and those bovine primary gastrointestinal epithelial-derived cells were successfully used to assess adherence and invasion of several intestinal pathogenic bacteria including enterohemorrhagic Escherichia coli
(EHEC) and Salmonella enterica serotype typhimurium. In addition, bovine colonic crypts cells isolated and purified from the mucosa, proved to be useful in vitro tools to study virulence factors of EHEC, verotoxins, in particular (10). The work showed the expression of globotriaosylceramide Gb3 by bovine colonocytes, which directly contrasts with the absence of this receptor on human intestinal epithelium. This fact represents a fundamental difference that could have major significance in the different pathogenicity of EHEC in these hosts, and make bovine colonocytes an invaluable tool for studying EHEC infection (10).

Bridger et al. (7) showed that epithelioid cells from bovine colonic crypts formed a confluent monolayer on the surface of collagen-coated culture flasks. Those bovine colonocytes expressed epithelial cell-specific cytokeratin and cell membraneassociated tight junctional ZO-1 in the contact area between neighboring cells. Semi-quantitative RT-PCR demonstrated variable amounts of gene transcripts for different TLR genes. Notably, primary bovine colonocytes expressed TLR4 mRNA while transcripts for TLR1, TLR3, and TLR6 were also detectable in some cultures. Moreover, the study showed that colonocytes significantly up-regulated the expression of IL-8, MCP-1, and RANTES when challenged with pathogenic E. coli or lipopolysaccharide (LPS) (7). Therefore, short-term bovine colonocytes cultures proved to be suitable in vitro models to study pathogens-specific responses of the bovine colonic mucosa.

Those studies clearly demonstrated that bovine primary IEC cultures represent valuable tools to assess the molecular mechanisms involved in pathologies caused by infectious agents. However, the cellular and molecular interactions of commensal or probiotic bacteria with BIE cells have been less explored.

There is an increasing research in the use of immunobiotics to beneficially modulate the mucosal immune system in animals. Immunobiotic bacteria could be used for improving resistance against pathogens and decreasing intestinal inflammatorymediated tissue damage (13-17). In this regard, our laboratories and others' have conducted in vitro and in vivo studies utilizing different lactobacilli and bifidobacteria strains to evaluate the effect of immunobiotics against infections and inflammation in animals, however, the majority of these studies were performed in swine and only few in the cattle (13).

We hypothesized that a cell line of BIE cells could be a useful in vitro model for the evaluation of the molecular interactions between IECs and bovine pathogens. In addition, such system would allow the selection of immunobiotic microorganisms and the study of the mechanisms by which probiotic lactic acid bacteria (LAB) functionally modulate bovine IECs. Therefore, in order to: (a) understand the functional role of IECs in bovine mucosal host defense and (b) select potential immunobiotic LAB strains that may be used to beneficially modulate the inflammatory response in bovine IECs; we have recently established an immortalized BIE cell line (18). Monolayer cobblestone and epithelial-like morphology is assumed by BIE cells when cultured, with close contact between the cells. Moreover, scanning electron microscopy examination of BIE cell reveled that 3-days old cells have irregular and slender microvilli-like structures on their surface and that this structures increase in complexity as the cells grow (18). BIE 
cells undergoes over at least 40 passages with no detectable loss of epithelial properties.

Expression of TLRs mRNA in BIE cells was evaluated by RTqPCR and it was demonstrated that all TLRs genes were expressed in BIE cells (19). TLR1, 3, 4, and 6 were strongly expressed, followed by TLR5, 8, 9, 10, 2, and 7. We were particularly interested in expression of TLR2 and TLR4 in BIE cells as the main receptors detecting $\operatorname{Gram}(+)$ probiotic bacteria and $\operatorname{Gram}(-)$ pathogens, respectively. Therefore, to confirm real-time PCR findings, we further examined the expression of TLR2 and 4 proteins in BIE cells using anti-TLRs antibodies that are able to cross-react with bovine TLRs (19). Visualization of the immunofluorescence staining confirmed the protein expression of TLR2 and 4 in BIE cells (Figure 1A).
A

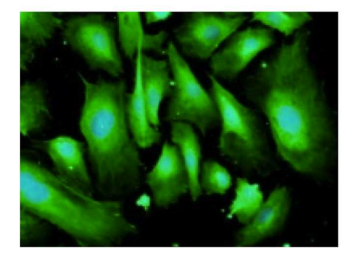

TLR4 $^{+}$BIE cells

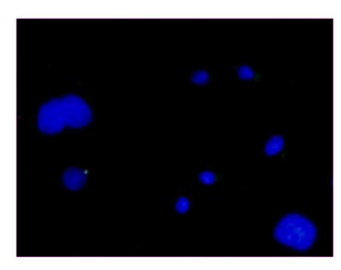

Isotype control BIE cells
$\mathbf{B}$

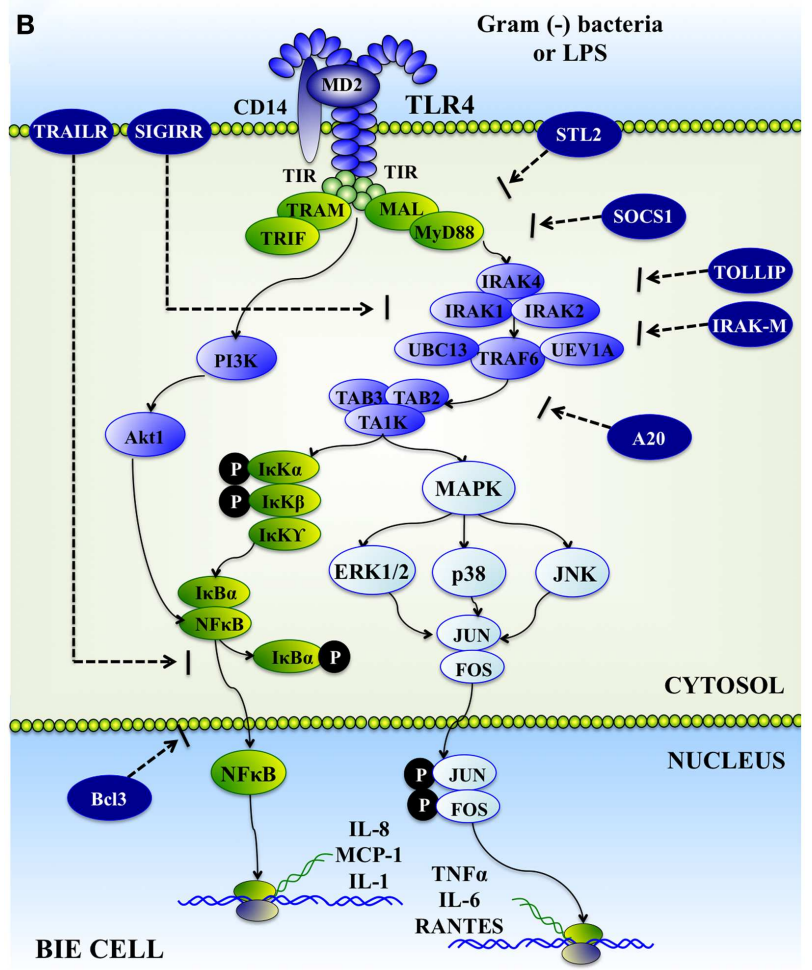

FIGURE 1 | Toll-like receptor 4 (TLR4) expression in bovine intestinal epithelial epitheliocytes (BIE cells) (A). Upon recognition of lipopolysaccharide (LPS), TLR4 dimerizes and initiates a signaling cascades that include phosphatidylinositol 3-kinase (PI3K), mitogen-activated protein kinase (MAPK), and nuclear factor $\kappa \mathrm{B}(\mathrm{NF}-\kappa \mathrm{B})$ pathways; and culminates in the production of inflammatory mediators by BIE cells (B).
The inflammatory response triggered by BIE cells in the face of a challenge with heat-stable enterotoxigenic E. coli (ETEC) MAMPs was also evaluated. Upon pathogen binding to TLR4 complex, this receptor recruits, through its short intracellular toll-interleukin1 receptor (TIR) domain, adaptor molecules, and kinases, thus initiating a downstream signaling cascade that culminates in the production and secretion of inflammatory mediators such as TNF$\alpha$, IL-1 $\beta$, IL-6, and IL-8 (Figure 1B). The ETEC 987P strain used in our experiments does not express the TLR5-ligand flagellin, and we demonstrated that the main molecule responsible for the inflammatory response triggered by this bacterium is the LPS (15, 16). Stimulation of BIE cells with heat-stable ETEC MAMPs from strain $987 \mathrm{P}$ enhanced the production of the pro-inflammatory cytokines IL- 6 , IL- 8 , IL- $1 \beta$, and MCP- 1 by activating mitogenactivated protein kinase (MAPK) and nuclear factor $\kappa \mathrm{B}(\mathrm{NF}-\kappa \mathrm{B})$ pathways (19). These findings are in line with our previous reports demonstrating that the heat-killed ETEC 987P strain triggers a TLR4-mediated inflammatory response in porcine intestinal epithelial (PIE) cells through NF- $\kappa$ B and MAPK pathways (20). In addition, our results in BIE cells correlate with studies of the immune response against ETEC in IECs of different hosts' species. It was shown that both NF- $\mathrm{B}$ and MAPK pathways are important mediators of ETEC and LPS activation in human (HT-29 and T84) and mouse (CMT93) IECs $(15,21)$.

Available lines of evidence indicate that bovine epithelial cells, including intestinal, mammary, bronchial, and nasopharynx epithelial cells respond to bacterial LPS and other microbial products by producing pro-inflammatory cytokines required to combat invading pathogens. Therefore, the pro-inflammatory mediators produced by BIE cells in response to ETEC may have an important protective role during the course of intestinal infections. The chemokine IL-8 stimulates a strong infiltration of neutrophils in the gut lamina propria, a fact that is consistently observed upon ETEC infection. After IL-8 induced recruitment of neutrophils, increase of IL-6 production is able to induce degranulation of these cells, thereby enhancing the inflammatory response (22). In addition, IECs are able to produce MCP-1 in response to ETEC challenge. This chemokine has potent monocyte-activating and attracting properties and plays a major role during intestinal inflammation (23). Therefore, BIE cells respond to the presences of ETEC and LPS by activating the TLR4-signaling pathway, which is necessary to initiate a robust defensive action against intruders.

Our studies indicate then that the BIE cell line could be a useful cell line for evaluating inflammatory responses via TLR4 in vitro. Furthermore, considering that inflammatory responses induced by intestinal pathogens can lead to dysregulation of IECs signaling, disruption of membrane barrier integrity, enhancement of pathogen translocation and disease (24), BIE cells could be also used to evaluate therapies designed for preventing inflammatory damage caused by bovine intestinal pathogens or their associated PAMPs or virulence factors.

\section{PROBIOTICS FOR THE BOVINE HOST}

Several studies on the pathogenesis of intestinal inflammation/infection both in man and experimental animals continue to show the importance of commensal bacteria in the gastrointestinal tract in stimulating and directing the immune system. Moreover, 
the ability of immunobiotic bacteria to beneficially modulate the response against intestinal pathogens in animals through the improvement of resistance and the reduction of inflammatorymediated tissue damage has been described by several reports (25-27). Before weaning, dairy calves are highly susceptible to several pathogens. For several years, antibiotics have been used to overcome these problems also to obtain economic benefits in terms of improved calves performance and reduced medication costs. However, the use of antibiotics in animal husbandry is in question because of antibiotic resistance of microorganisms. In an effort to replace antibiotics from bovine feeds, many additives have been proposed including the use of probiotics $(28,29)$. In fact, some few studies have shown that probiotic bacteria can be used as growth promoters in calves instead of antibiotics to counteract the negative effects of their widespread use (30). Early studies of Abe et al. (31) showed that oral administration of Bifidobacterium pseudolongum or Lactobacillus acidophilus to calves improved body weigh gain and decreased the frequency of diarrhea occurrence. Similarly, Mokhber-Dezfouli et al. (32) demonstrated that probiotic treatments have the ability to beneficially modulate body weight gain, body height, and general health condition of calves. Additionally, oral treatment with probiotic E. coli significantly reduced the pathogenicity and fecal shedding of EHEC in calves $(33,34)$. It was also reported that a mixture composed of Lactobacillus casei DSPV 318T, Lactobacillus salivarius DSPV 315T, and Pediococcus acidilactici DSPV 006T protected calves against Salmonella Dublin infection (35). These studies clearly show the potential of probiotic bacteria to beneficially modulate gastrointestinal hemostasis in the bovine host. However, the cellular and molecular mechanisms involved in the probiotic activities in cattle have not been studied in depth.

\section{REGULATION OF INFLAMMATION IN BIE CELLS BY IMMUNOBIOTIC LACTOBACILLI}

The first contact of immunobiotic bacteria with the intestinal mucosa is mediated by the single cell layer of IECs. As mentioned before, these IECs are of paramount importance in hostimmunobiotic cross-talk. Then, we thus sought to determine whether an immunobiotic Lactobacillus strain could regulate the inflammatory response induced by heat-stable ETEC MAMPs in BIE cells. Our previous studies with the strain Lactobacillus jensenii TL2937 showed that this bacterium has remarkable immunomodulatory effects in porcine IECs and immune cells [for a review see Ref. (3)]. The TL2937 strain is able to functionally modulate porcine IECs by inhibiting excessive MAPK- and NF- $\kappa$ Binduced pro-inflammatory cytokine production (IL-6 and IL-8) in response to TLR4 activation $(3,15)$. Consequently, we first focused on L. jensenii TL2937 to evaluate its anti-inflammatory effect in BIE cells. Preincubation of BIE cells with L. jensenii TL2937 significantly decreased IL-6 and IL-8 expressions in 20 and $25 \%$ with respect to the control, respectively, after heat-stable ETEC MAMPs challenge (19). However, this effect was lower when compared with the anti-inflammatory activity of this strain in PIE cells (15). In porcine, IECs previously treated with the TL2937 strain, stimulation with heat-stable ETEC MAMPs reduced IL- 6 and IL- 8 expressions by 35 and $30 \%$ when compared to control cells, respectively (15). Although the effect of the L. jensenii TL2937 in BIE cells was lower than the one previously reported for porcine IECs, our first studies in BIE cells indicated that probiotic lactobacilli could be beneficial for attenuating inflammatory damage caused by TLR4 activation in bovine epithelial cells (19). Thus, we next aimed to screen and select the most effective immunoregulatory lactobacilli strains able to modulate TLR4-mediated pro-inflammatory response in BIE cells. Several lactobacilli strains were evaluated in our bovine IECs line and we found that some of these bacteria were capable to downregulate the expression of inflammatory cytokines. Among these strains, L. casei OLL2768 showed the most pronounced effect (19). Notably, the anti-inflammatory activity of the OLL2768 strain was more pronounced than that observed for L. jensenii TL2937 in BIE cells, while the effect of OLL2768 strain was lower in PIE cells (15). It is well known that probiotic activities are strain specific. In addition, our findings clearly indicated that is necessary to carefully evaluate different strains according to the specific host, because the effect of the same Lactobacillus may be different according to the host that consumes it. Then, our in vitro bovine system could be of great value to find potential immunobiotic strains suitable for the improvement of the bovine host health.

We also aimed to define the molecular mechanisms by which L. casei OLL2768 attenuated heat-stable ETEC MAMPs-induced pro-inflammatory response in BIE cells. Our data showed that the immunoregulatory effect was related to the capacity of OLL2768 strain to inhibit NF- $\kappa$ B and MAPK p38 signaling pathways in bovine IECs after TLR4 activation.

Nuclear factor $\kappa \mathrm{B}$ is composed of several protein subunits regulating DNA transcription. Under non-stimulatory conditions, it is bound to the inhibitor molecule IkB in the cytoplasm. After TLR activation IkB is phosphorylated by IKK and once freed from IkB, NF- $\kappa$ B subunit p65 (RelA) migrates into the nucleus, where it binds to target promoters and activates transcription of effector genes including TNF- $\alpha$, IL- 8 , and others $(36,37)$. Among many up-stream signaling proteins involved in NF- $\kappa \mathrm{B}$ activation, TLR4 plays a critical role and it is well-documented that TLR4/NF- $\mathrm{B}$ pathway has a pivotal role in the pathogenesis of several intestinal inflammatory diseases and infection-induced tissue damage (38). Some studies have reported the ability of probiotic lactobacilli to modulate TLR4/NF- $\kappa \mathrm{B}$ pathway in the gut $(39,40)$. It was showed that Lactobacillus suntoryeus, a gut commensal, blocks inflammatory mediators (Cox2, TNF- $\alpha$, IL-1, and IL-6) through suppression of TLR4-linked NF- $\kappa \mathrm{B}$ activation in mice with 2,4,6trinitrobenzene sulfonic acid-induced colitis (41). Liu et al. (42) reported that Lactobacillus reuteri strains DSM 17938 and ATCCPTA4659 led to decrease intestinal protein levels of TLR4 and decreased pro-inflammatory cytokine levels in parallel with inhibition of TLR4-signaling via the NF- $\kappa \mathrm{B}$ pathway in newborn rats with necrotizing enterocolitis. In addition, it was reported that some probiotics strains are able to suppress TNF- or Salmonella typhimurium-induced IL- 8 gene expression and secretion by IECs in a NF- $\kappa \mathrm{B}$-dependent manner $(39,40)$. Our experiments also demonstrated that L. casei OLL2768 is able to inhibit TLR4/p38 signaling pathway since we demonstrated that in lactobacillitreated BIE cells the phosphorylation of p38 was reduced after challenge in heat-stable ETEC MAMPs (19). Regulation of MAPK p38 pathway by probiotics has been described before. L. rhamnosus 
GG was found to significantly down-regulate expression of p38 in human monocyte-derived DCs after the challenge with LPS (43). It was also showed that Lactobacillus bulgaricus LBG, inhibited the activation of the TLR4-signaling pathway and IL-8 production induced by Helicobacter pylori LPS in human gastric adenocarcinoma cells through blocking MAPK p38 (44). Then, our findings in BIE cells are reminiscent of other studies showing that probiotic L. casei OLL2768 is capable of modulating TLR4/NF- $\kappa \mathrm{B}-$ and TLR4/p38-induced inflammation.

The JNK and p38 MAPK pathways share several up-stream regulators, and accordingly there are multiple stimuli that simultaneously activate both pathways. In this regard, it was showed that the conditioned media from probiotic L. rhamnosus GG induced the expression of cellular heat shock protein (Hsp72) in IECs in a p38- and JNK-dependent manner (45). The work showed that L. rhamnosus GG conditioned media treatment resulted in a clear activation of both p38 and JNK pathways in IECs. Moreover, exposure of IECs to inhibitors against p38 and JNK before conditioned media treatment resulted in blockade of Hsp72 expression, thus, confirming a likely role for both MAPK signaling pathways in the probiotic effect (45). Then, we expected that L. casei OLL2768 had an inhibitory effect on JNK pathway in BIE cells as observed for the p38 MAPK pathway. However, increased levels of p-JNK were detected in BIE cells stimulated with the OLL2768 strain. It was also reported that JNK and p38 MAPK pathways may induce opposite effects. In fact, there is evidence indicating that the p38 MAPK pathway can negatively regulate JNK activity in several contexts $(46,47)$. The first evidence of the interaction of these two pathways was the observation that inhibition of p38 strongly increased the activation of JNK (46). The work analyzed the effect of specific p38 MAPK inhibitors, SB202190 or SB203580, on JNK phosphorylation in A549 human lung alveolar epithelial cells, and found that inhibition of p38 MAPK could induce JNK activation due to a compensatory mechanism (46). In addition, it was showed that the p38 inhibitor SB203580 enhances the activation of JNK isoforms after the challenge of IECs with IL-1 $\beta$ or by LPS in macrophages (46). In line with those studies, the kinetic analysis of p38 and JNK phosphorylation in BIE cells showed an early upregulation of $\mathrm{p}-\mathrm{p} 38$ between 5 and $10 \mathrm{~min}$ after heat-stable ETEC MAMPs challenge that was followed by a down-regulation of $\mathrm{p}$ JNK between 10 and $20 \mathrm{~min}$ (19). Therefore, we can speculate that L. casei OLL2768 has a direct influence in p38 pathway while its effect in JNK is the result of the inhibition of $\mathrm{p} 38$ phosphorylation. Further research is needed to clarify completely the influence of $L$. casei OLL2768 in MAPK pathways in BIE cells.

Following TLR activation, there must be a checkpoint where TLR signaling is abolished and the system is returned to a normal physiological state to avoid a harmful response toward the host immune system. Regulatory factors able to modulate the duration and intensity of TLRs signals are therefore key components for the protection of the hosts (48). Several regulatory mechanisms have been described for TLRs including soluble and decoy factors, membrane-associated protein regulators, negative regulators of the adaptor complex, and microRNA (3). To assess the expression of these negative regulators of TLRs, we first cloned cDNAs corresponding to these proteins in the bovine (19). We demonstrated the expression of A20-binding inhibitor of nuclear factor kappa B activation 3 (ABIN-3); B-cell lymphoma 3-encoded protein (Bcl3); interleukin-1 receptor-associated kinase $M$ (IRAK-M); single immunoglobulin IL-1-related receptor (SIGIRR); toll interacting protein (Tollip); and mitogen-activated protein kinase 1 (MKP-1) in BIE cells (19). Consequently, the effect of L. casei OLL2768 on the expression of these negative regulators of the TLRs signaling was next evaluated. L. casei OLL2768 is able to up-regulate Tollip and Bcl-3 in BIE cells, and in this way to negatively regulate TLR4 signaling (19). Tollip is able to suppress the activity of IL1 receptor-associated kinase (IRAK), and inhibit TLR4-triggered NF- $\kappa$ B and MAPK signaling pathways $(49,50)$. It was showed that stimulation of IECs with a TLR ligand, such as LPS, induces a state of hyporesponsiveness through up-regulation of Tollip that limits pro-inflammatory responses triggered by a second challenge with the same or another TLR ligand (50). The expression of Tollip was reported in bovine mammary epithelial cells (51). The work showed that expression of Tollip was increased in response to LPS, suggesting that the bovine mammary epithelium possesses the necessary immune repertoires required to regulate TLR4 activation. Recently, it was demonstrated by Fu et al. (52) that Tollip is significantly up-regulated in bovine endometrial epithelial cells after the stimulation with LPS, and that this up-regulation of Tollip was necessary for the regulation of the overexpression of $\mathrm{NF}-\mathrm{\kappa B}$ and the protection against the inflammatory damage. On the other hand, the $\mathrm{Bcl}-3$ is a nuclear protein and member of the NF- $\kappa \mathrm{B}$ family. Bcl-3 is able to stabilize repressive NF- $\kappa \mathrm{B}$ homodimers in a DNA-bound state, and in this way prevents the binding of transcriptionally active dimers. Therefore, Bcl-3 functions as an inhibitor of NF- $\kappa \mathrm{B}$ activity. In recent years, a role of $\mathrm{Bcl}-3$ has been revealed in LPS tolerance via its ability to stabilize the p50 homodimer, and thus, has been identified as a negative regulator of TLR4 signaling (53). Furthermore, by selectively affecting chromatin remodeling, $\mathrm{Bcl}-3$ mediates repression of pro-inflammatory genes, and also facilitates the expression of the anti-inflammatory gene $I L-10$ (54). Therefore, the induction of Bcl-3 and Tollip by $L$. casei OLL2768 in BIE cells is important in establishing tolerance against heat-stable ETEC MAMPs.

It is not possible to give a precise molecular mechanism for the anti-inflammatory action of L. casei OLL2768 on BIE cells at present. However, it could be hypothesized that interaction of L. casei OLL2768 with BIE cells through one or more PRRs induces the up-regulation of the negative regulators $\mathrm{Bcl}-3$ and Tollip, which reduce the production of inflammatory mediators in response to heat-stable ETEC MAMPs (Figure 2). One of the possible PRR involved in L. casei OLL2768 immunoregulatory capacities could be TLR2. Studies with the TLR2 ligand Pam3CSK4 in BIE cells demonstrated that the treatment with the TLR2 agonist up-regulate the expression of Tollip and reduce activation of NF- $\mathrm{B}$ and p38 MAPK pathways (19). However, further research is needed to resolve which PRR is activated by L. casei OLL2768 for the induction of negative regulators.

\section{REGULATION OF INFLAMMATION IN BIE CELLS BY IMMUNOBIOTIC BIFIDOBACTERIA}

Members of the genus Bifidobacterium are considered to be important constituents of the microbiota of animals, from insects to mammals. They are gut commensals extensively used by the 


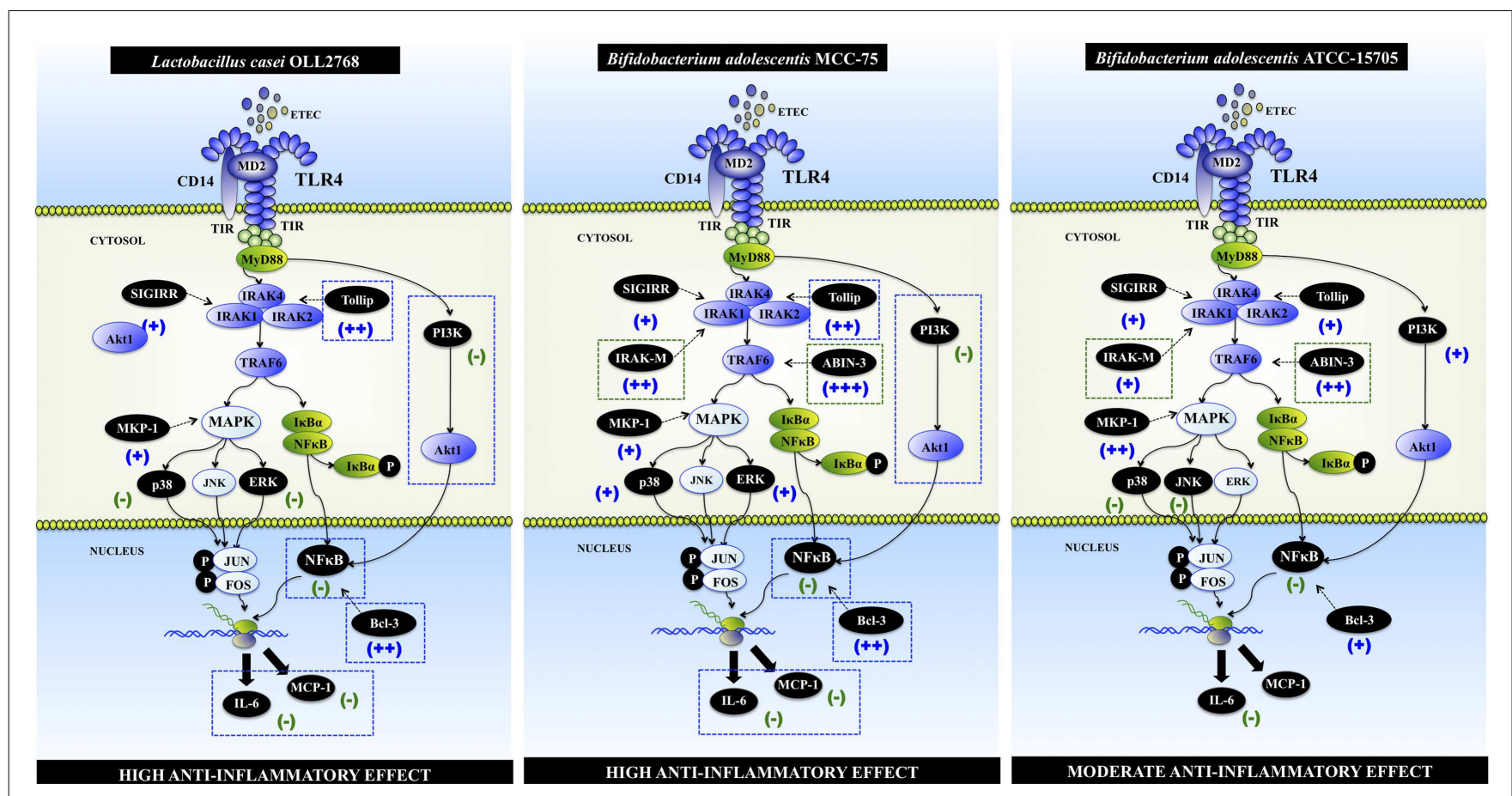

FIGURE 2 | Modulation of toll-like receptor 4 (TLR4) signaling pathway by Lactobacillus casei OLL2768, Bifidobacterium adolescentis MCC-75, and Bifidobacterium adolescentis ATCC15705 in bovine intestinal

epithelial epitheliocytes (BIE cells). Anti-inflammatory immunobiotic strains up-regulate the expression of TLR negative regulators, reduce the activation phosphatidylinositol 3-kinase (PI3K), mitogen-activated protein kinase (MAPK), and nuclear factor $\mathrm{KB}$ (NF-KB) pathways; and diminish the production of inflammatory mediators by BIE cells. food industry as probiotic microorganisms, since some strains have been shown to have specific beneficial effects. Bifidobacteria are able to prevent or alleviate infectious diarrhea through their effects on the immune system and resistance to colonization by pathogens. In addition, some bifidobacteria strains have potent anti-inflammatory capacities that could be used to reduce inflammatory-intestinal damage. Bifidobacterium animalis strain AHC7 decrease NF- $\kappa$ B activation in mice infected with S. typhimurium. B. animalis AHC7 consumption in this mouse model was associated with protection against inflammatory damage through modulation of secreted IL-10 and IL-12p70 and enhancement of Foxp3 expression in naïve T cells (55). In line with these results, it was showed that Bifidobacterium bifidum W23 was able to induce a suppression of IL- 8 synthesis by Caco- 2 cells challenged with $S$. enterica serovar enteritidis, and that the protective role of this probiotic strain was mediated, at least in part, via Hsp70 expression (56). Moreover, it was recently reported that Bifidobacterium adolescentis FRP 61, Bifidobacterium longum FRP 68 and FRP 69, and Bifidobacterium breve FRP 334 significantly reduced IL-8 production by HT-29 cells challenged with S. typhimurium (57). Others studies evaluating the effect of bifidobacteria in intestinal Caco- 2 cells showed that $B$. animalis MB5 avoid cytokine deregulation upon ETEC challenge by inducing upregulation of IL- $1 \beta$ and TNF- $\alpha$, and the down-regulation of TGF- $\beta$ expression $(58,59)$. Additionally, we demonstrated in porcine IECs cells that treatment with $B$. breve MCC-117 significantly reduced the expression of inflammatory cytokines in response to heat-stable ETEC
MAMPs. Moreover, studies with porcine immune cells showed that $B$. breve MCC-117 was able to reduce the levels of IFN$\gamma$ in $\mathrm{CD}^{+}$and $\mathrm{CD}^{+}$lymphocytes and improved IL-10 levels in $\mathrm{CD} 4{ }^{+} \mathrm{CD} 25^{\text {high }}$ Foxp $3^{+}$lymphocytes (14). These are among several other studies that clearly showed that bifidobacteria are highly effective in regulating pathogenic inflammation in the gut. Therefore, we next aimed to select potential immunomodulatory bifidobacteria able to beneficially modulate the inflammatory response in BIE cells.

The potential use of bifidobacteria as probiotic for cattle is supported by some new reports indicating the presence of these bacteria in young calf intestines and the fact that their presence in high numbers is associated with good health status of the host (60). Therefore, some bifidobacteria strains, previously selected in our porcine systems, were used to evaluate their anti-inflammatory capacities in heat-stable ETEC MAMPs-challenged-BIE cells (61). Similarly to the effect of $L$. casei OLL2768, some bifidobacteria were able to reduce the production of inflammatory mediators triggered by TLR4 activation in BIE cells. Considering their ability to reduce the expression of IL-6 and MCP-1, bifidobacteria strains were divided in the following two groups: (1) strains able to reduce both IL-6 and MCP-1 (B. adolescentis MCC-75 and $B$. breve MCC-117) and (2) strains able to reduce only IL-6 (B. longum BB536, B. adolescentis ATCC15705 and Bifidobacterium infantis MCC-1021) (61).

As described for the immunoregulatory L. casei OLL2768 strain, we also aimed to evaluate signaling pathways and TLR 
negative regulators expression in BIE cells after the treatment with bifidobacteria belonging to the two functional groups defined by our studies. Then, we selected B. adolescentis MCC-75, B. breve MCC-117 (strains with high anti-inflammatory capacities), and $B$. adolescentis ATCC15705 (strain with moderate anti-inflammatory capacity) for further experiments. Activation of MAPK, NF- $\kappa$, and phosphatidylinositol 3-kinase (PI3K) pathways, and changes in the expression of TLR negative regulators in MCC-75-, MCC117- and ATCC15705-treated BIE cells were then studied. We found that each bifidobacteria strain induces unique changes in TLR4 signaling in bovine IECs (61) (Figure 2).

As mentioned before, several negative regulatory mechanisms control TLRs-mediated inflammatory responses and restore immune system balance in the gut. Although the NF-кBdependent gene expression is critical to the induction of an efficient immune response, excessive, or prolonged NF- $\kappa \mathrm{B}$ signaling can contribute to the development of several inflammatory diseases. Therefore, this signaling transduction pathway has to be tightly regulated by several intracellular proteins. The ubiquitinediting enzyme A20 is key regulator of the TLRs signaling. It was showed that A20 deficiency in IECs renders mice sensitive to TNF$\alpha$-induced lethal inflammation $(62,63)$. Moreover, it was reported that A20 is an early response negative regulator of TLR4 and TLR5 signaling in IECs that functions during intestinal inflammation to control the innate immune system (64). In addition, the A20binding inhibitor of NF- $\kappa \mathrm{B}$ activation (ABIN) is LPS-inducible proteins that negatively regulate NF- $\kappa \mathrm{B}$ activation in response to TNF- $\alpha$ and LPS (65). ABINs have been described as three different proteins (ABIN-1, -2, and -3) that bind A20. Overexpression of ABINs inhibits NF- $\kappa$ B activation by TNF- $\alpha$ and several other stimuli. Similar to A20, ABIN-3 expression is NF- $\kappa$ B dependent, implicating a potential role for the A20/ABIN complex in the negative feedback regulation of NF- $\kappa \mathrm{B}$ activation (66). Therefore, the induction of A20/ABIN complex by bifidobacteria in BIE cells is important in establishing tolerance against heat-stable ETEC MAMPs. This is in line with our previous reports in porcine IECs. In our works in the porcine systems, we showed that the bifidobacteria strains with the highest capacity to downregulate the expression of inflammatory cytokines in response to heat-stable ETEC PAMPs were also able to up-regulate A20. In fact, the most potent anti-inflammatory bacteria evaluated in our laboratory, bifidobacteria strains BB536 and M-16V and L. jensenii TL2937, strongly up-regulated the ubiquitin-editing enzyme A20 [for a review see Ref. (3)].

$\mathrm{Bcl}-3$ protein functions as an inhibitor of NF- $\kappa \mathrm{B}$ activity as mentioned before. In addition, SIGIRR, Tollip, and IRAK-M are also known to be expressed at high levels in IECs, and to thereby contribute to the hyporesponsiveness of IECs to commensals (64, $67,68)$. Therefore, induction of these five negative regulators by bifidobacteria in BIE cells may be important for establishing tolerance against heat-stable ETEC MAMPs (Figure 2). Moreover, the fold expression increase of the negative regulators of the TLRs signaling should be also important since the levels of ABIN-3, IRAK-M, and Bcl-3 were significantly higher in B. breve MCC117- and B. adolescentis MCC-75-treated BIE cells when compared with BIE cells treated with the moderate anti-inflammatory strain B. adolescentis ATCC15705 (61).
On the other hand, the MAPK pathway is involved in the upregulation of several inflammatory genes, and MKP-1 plays a role in the inhibition of pro-inflammatory mRNA expression, because it can inactivate MAPK pathway (69). Therefore, we expected that bifidobacteria with high anti-inflammatory activity significantly up-regulate MKP-1 expression and reduce MAPK activation as we have observed with other anti-inflammatory immunobiotic strains (3). However, when ERK, p38, and JNK MAPK activation and MKP-1 expression were studied in BIE cells treated with bifidobacteria, we found that B. adolescentis MCC-75 and B. breve MCC-117 activated ERK MAPK pathway and only moderately up-regulated MKP-1. On the contrary, B. adolescentis ATCC15705 strongly increased expression of MKP-1 and inhibit p38 and JNK pathways (61). It is known that the ERK pathway play key regulatory functions in a diverse spectrum of biological processes such as cell proliferation, differentiation, survival, and motility (70). It was also reported that TGF- $\beta$ induces ERK activity in IECs and this TGF- $\beta / E R K$ interaction regulates genes that are crucial for cell growth, migration, and survival of IECs $(71,72)$. In fact, treatment with TGF- $\beta$ prevents mucosal-injury, enhances $\mathrm{p}$-ERK and $\beta$-catenin, induces enterocyte proliferation, inhibits enterocyte apoptosis, and improves intestinal recovery following methotrexate-induced intestinal-mucositis in rats (73). Moreover, TGF- $\beta$ increases protein levels, collagen I, TGF- $\beta$ of type- 1 inhibitor of plasminogen activator, and the TGF- $\beta$-converting enzyme furin in various IEC lines via ERK (74) indicating an important immunoregulatory role of the ERK pathway in maintaining homeostasis in IECs. Therefore, the activation of the ERK pathway by B. adolescentis MCC-75 and B. breve MCC-117 during ETEC MAMPs-mediated inflammation could have an important protective role against inflammatory damage (61).

Our studies also demonstrated that both B. adolescentis MCC75 and $B$. breve MCC-117 were able to inhibit PI3K pathway in heat-stable ETEC MAMPs-challenged-BIE cells. It is known that PI3K regulates TLR signaling in both positive and negative ways. By mutating specific tyrosine residues in the cytosolic domain of TLR2, it was showed that there is a loss in the capacity of p85 to associate with this receptor and in the ability of TLR2 to activate NF- $\mathrm{B}$ pathway. Furthermore, inhibition of PI3K during TLR2 stimulation has been shown to reduce NF- $\kappa$ B activation (75). On the contrary, studies in PI3K or p85a deficient mice showed that PI3K negatively regulates TLR signaling $(76,77)$. Then, it is well established that PI3K could affect TLRs signaling pathways in different ways and that it effect depends on cell type and readout. In our studies, we showed that stimulation of BIE cells with heat-stable ETEC MAMPs activated PI3K pathway, indicating that PI3K is positively involved in TLR4 signaling in BIE cells (Figure 1B). Moreover, we demonstrated that bifidobacteria able to reduce activation PI3K pathway were the strains with the highest anti-inflammatory activity (61) (Figure 2).

As mentioned before, our works evaluating the immunoregulatory activity of immunobiotics demonstrated that the upregulation of some regulatory cytokines and down-regulation of inflammatory mediators is dependent of TLR2 activation (15, 16). Therefore, we also investigated the role of TLR2 in the immunoregulatory effects of $B$. adolescentis MCC-75, B. breve MCC-117, and B. adolescentis ATCC15705 by using anti-TLR2 
blocking antibodies (61). It was showed that the reduction of IL-6 induced by bifidobacteria in ETEC MAMPs-challenged-BIE cells was abolished when anti-TLR2 antibodies were used. This is in line with other reports conferring a key role to TLR2 in the recognition of bifidobacteria, which possess anti-inflammatory activities (7880 ). It is known that stimulation of TLR 2 is able to induce tolerance against a subsequent LPS challenge (81). Therefore, it is possible that bifidobacteria could induce this type of cross-tolerance in BIE cells through their interaction with TLR2. In addition, we showed that the reduction of MCP-1 levels after challenge of BIE cells was not abolished when anti-TLR2 antibodies were used. This finding indicates that additional PRRs may be involved in the anti-inflammatory effects of B. adolescentis MCC-75, and B. breve MCC-117 in BIE cells.

\section{CONCLUSION}

The knowledge of the cellular and molecular interactions of human IECs with commensal and probiotic bacteria is rapidly progressing. An exciting possibility is that similar systems developed for the bovine host could serve as a platform for medicine and research. However, to achieve this goal, bovine IECs cultures must be enhanced and improved to allow that functional assays can be performed. Our research work has demonstrated that the BIE cell line is a useful in vitro tool for the study of TLR4-induced inflammatory responses in the bovine intestinal epithelium. We have also demonstrated that BIE cells could be used for a rapid screening and selection of potential immunobiotic bacteria as well as for studying the molecular mechanisms involved in their beneficial protective activity.

Despite the unique effect of each lactobacilli or bifidobacteria strain, some general conclusions can be made when comparing the effect of the two different immunoregulatory groups: high anti-inflammatory activity (L. casei OLL2768, B. adolescentis MCC-75, and B. breve MCC-117) and moderate antiinflammatory activity (B. adolescentis ATCC15705) (Figure 2): (1) anti-inflammatory capacity in BIE cells is strain dependent, as demonstrated by the differential effect induced by each strain, even those of the same specie (B. adolescentis MCC-75 and B. adolescentis ATCC15705). (2) The upregulation of TLR negative regulators and the intensity of that upregulation would be related to the different immunomodulatory capacity of each immunobiotic strain. Notably, upregulation of Tollip and Bcl-3 seems to be related to a high anti-inflammatory capacity. (3) The inhibition of PI3K pathway would be related to the high anti-inflammatory effect of immunobiotics in BIE cells. (4) The balance between MAPK activation and MKP-1 upregulation would be related to the anti-inflammatory effect of bifidobacteria in BIE cells. (5) The anti-inflammatory effect of immunobiotics in BIE cells is partially dependent on TLR2. Further research is needed to resolve which other PRR is involved in the immunoregulatory effects. In addition, one general conclusion can be made when comparing the effect of the two different immunoregulatory groups of bifidobacteria (Figure 2). (6) The upregulation of IRAK-M and ABIN-3 and the intensity of that upregulation would be related to the different immunomodulatory capacity of each bifidobacteria strain.

We believe that studies in BIE cell would provide useful information that may help in the near future to develop new functional feeds able to beneficially modulate the mucosal immune system in the bovine host. In line with this, we showed in our studies that the immunoregulatory strains L. casei OLL2768, B. adolescentis MCC75 , and $B$. breve MCC- 117 are able to functionally modulate BIE cells by attenuating TLR4-induced NF- $\kappa$ B, MAPK, and IP3K activation and inflammatory cytokines production. Then, OLL2768, MCC-75, and MCC-117 strains would be good candidates for in vivo evaluation of the protective effect of immunobiotics against inflammatory damage induced by bovine intestinal pathogens or their associated PAMPs. We also believe that the immunobiotic application in cattle could contribute to produce safety animal foods via improving bovine health.

\section{ACKNOWLEDGMENTS}

This study was partially supported by a Grant-in-Aid for Scientific Research (B) (No. 24380146) and Challenging Exploratory Research (No. 26660216) from the Japan Society for the Promotion of Science (JSPS), and the Japan Dairy Association (J-milk) to Dr. Haruki Kitazawa. We thank Leonardo Albarracin for his help with the design and development of figures.

\section{REFERENCES}

1. Yen TH, Wright NA. The gastrointestinal tract stem cell niche. Stem Cell Rev (2006) 2:203-12. doi:10.1007/s12015-006-0048-1

2. Wells JM, Rossi O, Meijerink M, van Baarlen P. Epithelial crosstalk at the microbiota-mucosal interface. Proc Natl Acad Sci U S A (2011) 108:4607-14. doi:10.1073/pnas.1000092107

3. Villena J, Kitazawa H. Modulation of intestinal TLR4-inflammatory signalling pathways by probiotic microorganisms: lessons learned from Lactobacillus jensenii TL2937. Front Immunol (2014) 4:512. doi:10.3389/fimmu.2013.00512

4. Marco ML, Pavan S, Kleerebezem M. Towards understanding molecular modes of probiotic action. Curr Opin Biotechnol (2006) 17:204-10. doi:10.1016/j. copbio.2006.02.005

5. Clancy R. Immunobiotics and the probiotic evolution. FEMS Immunol Med Microbiol (2003) 38:9-12. doi:10.1016/S0928-8244(03)00147-0

6. Lebeer S, Vanderleyden J, De Keersmaecker CJ. Host interactions of probiotic bacterial surface molecules: comparison with commensals and pathogens. Nat Rev Microbiol (2010) 8:171-84. doi:10.1038/nrmicro2297

7. Bridger PS, Mohr M, Stamm I, Fröhlich J, Föllmann W, Birkner S, et al. Primary bovine colonic cells: a model to study strain-specific responses to Escherichia coli. Vet Immunol Immunopathol (2010) 137:54-63. doi:10.1016/j.vetimm.2010. 04.010

8. Dibb-Fuller MP, Best A, Stagg DA, Cooley WA, Woodward MJ. An in-vitro model for studying the interaction of Escherichia coli O157:H7 and other enteropathogens with bovine primary cell cultures. J Med Microbiol (2001) 50:759-69.

9. Föllmann W, Birkner S. The use of cultured primary bovine colon epithelial cells as a screening model to detect genotoxic effects of heterocyclic aromatic amines in the comet assay. J Toxicol Environ Health (2008) 71:947-53. doi:10.1080/15287390801988962

10. Hoey DEE, Sharp L, Currie C, Lingwood CA, Gally DL, Smith DGE. Verotoxin 1 binding to intestinal crypt epithelial cells results in localization to lysosomes and abrogation of toxicity. Cell Microbiol (2003) 5:85-97. doi:10.1046/j.14625822.2003.00254.x

11. Rusu D, Loret S, Peulen O, Mainil J, Dandrifosse G. Immuno-chemical, biomolecular and biochemical characterization of bovine epithelial intestinal primocultures. BMC Cell Biol (2005) 6:42. doi:10.1186/1471-2121-6-42

12. Baskaran S, Venkitanarayana A. Plant-derived antimicrobials reduce E. coli O157:H7 virulence factors critical for colonization in cattle gastrointestinal tract in vitro. Biomed Res Int (2014) 2014:212395. doi:10.1155/2014/212395

13. Gaggía F, Mattarelli P, Biavati B. Probiotics and prebiotics in animal feeding for safe food production. Int J Food Microbiol (2010) 141:S15-28. doi:10.1016/j. ijfoodmicro.2010.02.031

14. Fujie H, Villena J, Tohno M, Morie K, Simazu T, Aso H, et al. Toll-like receptor-2 activating bifidobacteria strains differentially regulate inflammatory cytokines in 
porcine intestinal epithelial cell culture system: finding new anti-inflammatory immunobiotics. FEMS Immunol Med Microbiol (2011) 63:129-39. doi:10.1111/ j.1574-695X.2011.00837.x

15. Shimazu T, Villena J, Tohno M, Fujie H, Hosoya S, Shimosato T, et al. Immunobiotic Lactobacillus jensenii elicit anti-inflammatory activity in porcine intestinal epithelial cells by modulating negative regulators of the toll-like receptor signaling pathway. Infect Immun (2012) 80:276-88. doi:10.1128/IAI.05729- 11

16. Villena J, Suzuki R, Fujie H, Chiba E, Takahashi T, Shimazu T, et al. Immunobiotic Lactobacillus jensenii modulates toll-like receptor 4-induced inflammatory response via negative regulation in porcine antigen presenting cells. Clin Vaccine Immunol (2012) 19:1038-53. doi:10.1128/CVI.00199- 12

17. Maldonado NC, Silva de Ruiz C, Otero MC, Sesma F, Nader-Macías ME. Lactic acid bacteria isolated from young calves - characterization and potential as probiotics. Res Vet Sci (2012) 92:342-9. doi:10.1016/j.rvsc.2011.03.017

18. Miyazawa K, Hondo T, Kanaya T, Tanaka S, Takakura I, Itani W, et al. Characterization of newly established bovine intestinal epithelial cell line. Histochem Cell Biol (2010) 133:125-34. doi:10.1007/s00418-009-0648-3

19. Takanashi N, Tomosada Y, Villena J, Murata K, Takahashi T, Chiba E, et al. Advanced application of bovine intestinal epithelial cell line for evaluating regulatory effect of lactobacilli against heat-killed enterotoxigenic Escherichia coli-mediated inflammation. BMC Microbiol (2013) 13:54. doi:10.1186/14712180-13-54

20. Lee JD, Mo JH, Shen C, Rucker AN, Raz E. Toll-like receptor signaling in intestinal epithelial cells contributes to colonic homoeostasis. Curr Opin Gastroenterol (2007) 23:27-31. doi:10.1097/MOG.0b013e3280118272

21. Cario E, Rosenberg IM, Brandwein SL, Beck PL, Reinecker HC, Podolsky DK. Lipopolysaccharide activates distinct signaling pathways in intestinal epithelial cell lines expressing toll-like receptors. J Immunol (2000) 164:966-72. doi:10.4049/jimmunol.164.2.966

22. Sitaraman SV, Merlin D, Wang L, Wong M, Gewirtz AT, Si-Tahar M, et al. Neutrophil-epithelial crosstalk at the intestinal lumenal surface mediated by reciprocal secretion of adenosine and IL-6. J Clin Invest (2001) 107:861-9. doi:10.1172/JCI11783

23. Warhurst AC, Hopkins SJ, Warhurst G. Interferon gamma induces differential up-regulation of alpha and beta chemokine secretion in colonic epithelial cell lines. Gut (1998) 42:208-13. doi:10.1136/gut.42.2.208

24. Berkes J, Viswanathan VK, Savkovic SD, Hecht G. Intestinal epithelial responses to enteric pathogens: effects on the tight junction barrier, ion transport, and inflammation. Gut (2003) 52:439-51. doi:10.1136/gut.52.3.439

25. Kitazawa H,Villena J. Modulation of respiratory TLR3-antiviral response by probiotic microorganisms: lessons learned from Lactobacillus rhamnosus CRL1505. Front Immunol (2014) 5:201. doi:10.3389/fimmu.2014.00201

26. Salva S, Villena J, Alvarez S. Diferential immunomodulatory activity of Lactobacillus rhamnosus strains isolated from goat milk: impact on intestinal and respiratory infections. Int J Food Microbiol (2010) 141:82-9. doi:10.1016/j. ijfoodmicro.2010.03.013

27. Villena J, Racedo S, Agüero G, Bru E, Medina M, Alvarez S. Lactobacillus casei improves resistance to pneumococcal respiratory infection in malnourished mice. J Nutr (2005) 135:1462-9.

28. Maldonado NC, de Ruiz CS, Otero MC, Sesma F, Nader-Macías ME. Lactic acid bacteria isolated from young calves - characterization and potential as probiotics. Res Vet Sci (2012) 92:342-9. doi:10.1016/j.rvsc.2011.03.017

29. Nader-Macías ME, Otero MC, Espeche MC, Maldonado NC. Advances in the design of probiotic products for the prevention of major diseases in dairy cattle. J Ind Microbiol Biotechnol (2008) 35:1387-95. doi:10.1007/s10295-008-0438-2

30. Signorini ML, Soto LP, Zbrun MV, Sequeira GJ, Rosmini MR, Frizzo LS. Impact of probiotic administration on the health and fecal microbiota of young calves: a meta-analysis of randomized controlled trials of lactic acid bacteria. Res Vet Sci (2012) 93:250-8. doi:10.1016/j.rvsc.2011.05.001

31. Abe F, Ishibashi N, Shimamura S. Effect of administration of bifidobacteria and lactic acid bacteria to newborn calves and piglets. J Dairy Sci (1995) 78:2838-46. doi:10.3168/jds.S0022-0302(95)76914-4

32. Mokhber-Dezfouli MR, Tajik P, Bolourchi M, Mahmoudzadeh H. Effects of probiotics supplementation in daily milk intake of newborn calves on body weight gain, body height, diarrhea occurrence and health condition. Pak J Biol Sci (2007) 10:3136-40. doi:10.3923/pjbs.2007.3136.3140

33. Zhao T, Tkalcic S, Doyle MP, Harmon BG, Brown CA, Zhao P. Pathogenicity of enterohemorrhagic Escherichia coli in neonatal calves and evaluation of fecal shedding by treatment with probiotic Escherichia coli. J Food Prot (2003) 66:924-30.

34. Tkalcic S, Zhao T, Harmon BG, Doyle MP, Brown CA, Zhao P. Fecal shedding of enterohemorrhagic Escherichia coli in weaned calves following treatment with probiotic Escherichia coli. J Food Prot (2003) 66:1184-9.

35. Frizzo LS, Zbrun MV, Soto LP, Bertozzi E, Sequeira GJ, Marti LE, et al. Pathogen translocation and histopathological lesions in an experimental model of Salmonella Dublin infection in calves receiving lactic acid bacteria and lactose supplements. J Vet Sci (2012) 13:261-70. doi:10.4142/jvs.2012.13.3.261

36. Haller D, Holt L, Parlesak A, Zanga J, Bauerlein A. Differential effect of immune cells on non-pathogenic Gram-negative bacteria-induced nuclear factor-kappaB activation and pro-inflammatory gene expression in intestinal epithelial cells. Immunology (2004) 112:310-20. doi:10.1111/j.1365-2567.2004.01874.x

37. Bruno ME, Frantz AL, Rogier EW, Johansen FE, Kaetzel CS. Regulation of the polymeric immunoglobulin receptor by the classical and alternative NF-kappaB pathways in intestinal epithelial cells. Mucosal Immunol (2011) 4:468-78. doi:10.1038/mi.2011.8

38. Andersen V, Christensen J, Ernst A. Polymorphisms in NF-кB, PXR, LXR, PPAR $\gamma$ and risk of inflammatory bowel disease. World J Gastroenterol (2011) 17:197-206. doi:10.3748/wjg.v17.i2.197

39. O’Hara AM, O'Regan P, Fanning A, Mahony C, Macsharry J, Lyons A, et al. Shanahan, functional modulation of human intestinal epithelial cell responses by Bifidobacterium infantis and Lactobacillus salivarius. Immunology (2006) 118:202-15. doi:10.1111/j.1365-2567.2006.02358.x

40. Zhang L, Li N, Caicedo R, Neu J. Alive and dead Lactobacillus rhamnosus GG decrease tumor necrosis factor-alpha-induced interleukin-8 production in Caco-2 cells. J Nutr (2005) 135:1752-6.

41. Lee JH, Lee B, Lee HS. Lactobacillus suntoryeus inhibits pro-inflammatory cytokine expression and TLR-4-linked NF-kappaB activation in experimental colitis. Int J Colorectal Dis (2009) 24:231-7. doi:10.1007/s00384-008-0618-6

42. Liu Y, Fatheree NY, Mangalat N, Rhoads JM. Lactobacillus reuteri strains reduce incidence and severity of experimental necrotizing enterocolitis via modulation of TLR4 and NF-кB signaling in the intestine. Am J Physiol Gastrointest Liver Physiol (2012) 302:G608-17. doi:10.1152/ajpgi.00266.2011

43. Giahi L, Aumueller E, Elmadfa I, Haslberger AG. Regulation of TLR4, p38 MAPkinase, I $\mathrm{B}$ and miRNAs by inactivated strains of lactobacilli in human dendritic cells. Benef Microbes (2012) 3:91-8. doi:10.3920/BM2011.0052

44. Zhou C, Ma FZ, Deng XJ, Yuan H, Ma HS. Lactobacilli inhibit interleukin8 production induced by Helicobacter pylori lipopolysaccharide-activated toll-like receptor 4. World J Gastroenterol (2008) 14:5090-5. doi:10.3748/wjg. 14.5090

45. Tao Y, Drabik KA, Waypa TS, Musch MW, Alverdy JC, Schneewind O, et al. Soluble factors from Lactobacillus GG activate MAPKs and induce cytoprotective heat shock proteins in intestinal epithelial cells. Am J Physiol Cell Physiol (2006) 290:C1018-30. doi:10.1152/ajpcell.00131.2005

46. Cheung PC, Campbell DG, Nebreda AR, Cohen P. Feedback control of the protein kinase TAK1 by SAPK2a/p38. EMBO J (2003) 22:5793-805. doi:10.1093/ emboj/cdg552

47. Muniyappa H, Das KC. Activation of c-Jun N-terminal kinase (JNK) by widely used specific p38 MAPK inhibitors SB202190 and SB203580: a MLK3-MKK7-dependent mechanism. Cell Signal (2008) 20:675-83. doi:10. 1016/j.cellsig.2007.12.003

48. Liew FY, Brint XD, EK O, Neill LA. Negative regulation of toll-like receptormediated immune responses. Nat Rev Immunol (2005) 5:446-58. doi:10.1038/ nri1630

49. Burns K, Clatworthy J, Martin L, Martinon F, Plumpton C, Maschera B, et al. Tollip, a new component of the IL-1RI pathway, links IRAK to the IL-1 receptor. Nat Cell Biol (2000) 2:346-51. doi:10.1038/35014038

50. Zhang G, Ghosh S. Negative regulation of toll-like receptor-mediated signaling by Tollip. J Biol Chem (2002) 277:7059-65. doi:10.1074/jbc.M109537200

51. Ibeagha-Awemu EM, Lee JW, Ibeagha AE, Bannerman DD, Paape MJ, Zhao X. Bacterial lipopolysaccharide induces increased expression of toll-like receptor (TLR) 4 and downstream TLR signaling molecules in bovine mammary epithelial cells. Vet Res (2008) 39:11. doi:10.1051/vetres:2007047

52. Fu Y, Liu B, Feng X, Liu Z, Liang D, Li F, et al. Lipopolysaccharide increases tolllike receptor 4 and downstream toll-like receptor signaling molecules expression in bovine endometrial epithelial cells. Vet Immunol Immunopathol (2013) 151:20-7. doi:10.1016/j.vetimm.2012.09.039 
53. Wessells J, Baer M, Young HA, Claudio E, Brown K, Siebenlist U, et al. Bcl-3 and NF-kB p50 attenuate lipopolysaccharide-induced inflammatoryresponses in macrophages. J Biol Chem (2004) 279:49995-50003. doi:10.1074/jbc. M404246200

54. Foster SL, Hargreaves DC, Medzhitov R. Gene-specific control of inflammation by TLR-induced chromatin modifications. Nature (2007) 447:972-8.

55. O’Mahony D, Murphy S, Boileau T, Park J, O’Brien J, Groeger G, et al. Bifidobacterium animalis $\mathrm{AHC7}$ protects against pathogen-induced NF- $\kappa \mathrm{B}$ activation in vivo. BMC Immunol (2010) 11:63. doi:10.1186/1471-2172-11-63

56. Malago JJ, Tooten PC, Koninkx JF. Anti-inflammatory properties of probiotic bacteria on Salmonella-induced IL-8 synthesis in enterocyte-like Caco- 2 cells. Benef Microbes (2010) 1:121-30. doi:10.3920/BM2009.0021

57. Carey CM, Kostrzynska M. Lactic acid bacteria and bifidobacteria attenuate the proinflammatory response in intestinal epithelial cells induced by Salmonella enterica serovar typhimurium. Can J Microbiol (2013) 59:9-17. doi:10.1139/cjm-2012-0446

58. Roselli M, Finamore A, Britti MS, Konstantinov SR, Smidt H, de Vos WM, et al. The novel porcine Lactobacillus sobrius strain protects intestinal cells from enterotoxigenic Escherichia coli K88 infection and prevents membrane barrier damage. J Nutr (2007) 137(12):2709-16.

59. Roselli M, Finamore A, Britti MS, Mengheri E. Probiotic bacteria Bifidobacterium animalis MB5 and Lactobacillus rhamnosus GG protect intestinal Caco-2 cells from the inflammation-associated response induced by enterotoxigenic Escherichia coli K88. Br J Nutr (2006) 95:1177-84. doi:10.1079/ BJN20051681

60. Bunešová V, Domig KJ, Killer J, Vlková E, Kopecný J, Mrázek J, et al. Characterization of bifidobacteria suitable for probiotic use in calves. Anaerobe (2012) 18:166-8. doi:10.1016/j.anaerobe.2011.09.008

61. Murata K, Villena J, Tomosada Y, Risa H, Chiba E, Shimazu T, et al. Bifidobacteria upregulate expression of toll-like receptor negative regulators counteracting enterotoxigenic Escherichia coli-mediated inflammation in bovine intestinal epitheliocytes. Open J Vet Med (2013) 3:143-55. doi:10.4236/ ojvm.2013.32023

62. Vereecke L, Sze M, McGuire C, Rogiers B, Chu Y, Schmidt-Supprian M, et al. Enterocyte-specific A20 deficiency sensitizes to tumor necrosis factorinduced toxicity and experimental colitis. J Exp Med (2010) 207:1513-23. doi:10.1084/jem.20092474

63. Shibolet O, Podolsky DK. TLRs in the gut. IV. Negative regulation of toll-like receptors and intestinal homeostasis: addition by subtraction. Am J Physiol Gastrointest Liver Physiol (2007) 292(6):G1469-73. doi:10.1152/ajpgi.00531. 2006

64. Oshima N, Ishihara S, Rumi MA, Aziz MM, Mishima Y, Kadota C, et al. A20 is an early responding negative regulator of toll-like receptor 5 signalling in intestinal epithelial cells during inflammation. Clin Exp Immunol (2010) 159(2):185-98. doi:10.1111/j.1365-2249.2009.04048.x

65. Verstrepen L, Adib-Conquy M, Kreike M, Carpentier I, Adrie C, Cavaillon JM, et al. Expression of the NF-kappaB inhibitor ABIN-3 in response to TNF and toll-like receptor 4 stimulation is itself regulated by NF-kappaB. J Cell Mol Med (2008) 12:316-29. doi:10.1111/j.1582-4934.2007.00187.x

66. Verstrepen L, Carpentier I, Verhelst K, Beyaert R. ABINs: A20 binding inhibitors of NF-Kappa B and apoptosis signaling. Biochem Pharmacol (2009) 78(2):105-11. doi:10.1016/j.bcp.2009.02.009

67. Xiao H, Gulen MF, Qin J, Yao J, Bulek K, Kish D, et al. The toll-interleukin-1 receptor member SIGIRR regulates colonic epithelial homeostasis, inflammation, and tumorigenesis. Immunity (2007) 26:461-475. doi:10.1016/j.immuni. 2007.02.012

68. Sugi Y, Takahashi K, Nakano K, Hosono A, Kaminogawa S. Transcription of the Tollip gene is elevated in intestinal epithelial cells through impaired OGlcNA-cylation-dependent nuclear translocation of the negative regulator Elf1. Biochem Biophys Res Commun (2011) 412(4):704-9. doi:10.1016/j.bbrc.2011. 08.035
69. Zhao Q, Wang X, Nelin LD, Yao Y, Matta R, Manson ME, et al. MAP kinase phosphatase 1 controls innate immune responses and suppresses endotoxic shock. J Exp Med (2006) 203(1):131-40. doi:10.1084/jem.20051794

70. Kohno M, Pouyssegur J. Targeting the ERK signaling pathway in cancer therapy. Ann Med (2006) 38(3):200-11. doi:10.1080/07853890600551037

71. Hartsough MT, Mulder KM. Transforming growth factor beta activation of p44MAPK in proliferating cultures of epithelial cells. J Biol Chem (1995) 270:7117-24. doi:10.1074/jbc.270.13.7117

72. Mamane Y, Petroulakis E, LeBacquer O, Sonenberg N. mTOR translation initiation and cancer. Oncogene (2006) 25:6416-22. doi:10.1038/sj.onc.1209888

73. Ben-Lulu S, Pollak Y, Mogilner J, Bejar J, G Coran A, Sukhotnik I. Dietary transforming growth factor-beta 2 (TGF- $\beta 2$ ) supplementation reduces methotrexateinduced intestinal mucosal injury in a rat. PLoS One (2012) 7:e45221. doi:10. 1371/journal.pone.0045221

74. Blanchette F, Rivard N, Rudd P, Grondin F, Attisano F, Dubois CM. CrossTalk between the p42/p44 MAP kinase and Smad pathways in transforming growth factor beta 1-induced furin gene transactivation. J Biol Chem (2001) 276:33986-94. doi:10.1074/jbc.M100093200

75. Arbibe L, Mira JP, Teusch N, Kline L, Guha M, Mackman N. Toll-like receptor 2-mediated NF-kappa B activation requires a Rac1-dependent pathway. Nat Immunol (2000) 1:533-40. doi:10.1038/82797

76. Fukao T, Tanabe M, Terauchi Y, Ota T, Matsuda S, Asano T. PI3K-mediated negative feedback regulation of IL-12 production in DCs. Nat Immunol (2002) 3:875-81. doi:10.1038/ni825

77. Yu Y, Nagai S, Wu H, Neish AS, Koyasu S, Gewirtz AT. TLR5-mediated phosphoinositide 3-Kinase activation negatively regulates flagellin-induced proinflammatory gene expression. J Immunol (2006) 176(10):6194-201. doi:10.4049/ jimmunol.176.10.6194

78. Hoarau C, Martin L, Faugaret D, Baron C, Dauba A, Aubert-Jacquin C, et al. Supernatant from Bifidobacterium differentially modulates transduction signaling pathways for biological functions of human dendritic cells. PLoS One (2008) 3(7):e2753. doi:10.1371/journal.pone.0002753

79. Otte JM, Podolsky DK. Functional modulation of enterocytes by Gram-positive and Gram-negative microorganisms. Am J Physiol Gastrointest Liver Physiol (2004) 286(4):G613-26. doi:10.1152/ajpgi.00341

80. Zeuthen LH, Fink LN, Frøkiaer H. Toll-like receptor 2 and nucleotide-binding oligomerization domain-2 play divergent roles in the recognition of gutderived Lactobacilli and Bifidobacteria in dendritic cells. Immunology (2008) 124(4):489-95. doi:10.1111/j.1365-2567.2007.02800.x

81. Zanello G, Meurens F, Berri M, Chevaleyre C, Melo S, Auclair E, et al. Saccharomyces cerevisiae decreases inflammatory responses induced by $\mathrm{F} 4+$ enterotoxigenic Escherichia coli in porcine intestinal epithelial cells. Vet Immunol Immunopathol (2011) 141(1-2):133-8. doi:10.1016/j.vetimm.2011.01.018

Conflict of Interest Statement: The authors declare that the research was conducted in the absence of any commercial or financial relationships that could be construed as a potential conflict of interest.

Received: 11 June 2014; accepted: 19 August 2014; published online: 02 September 2014. Citation: Villena J, Aso $H$ and Kitazawa $H$ (2014) Regulation of toll-like receptors-mediated inflammation by immunobiotics in bovine intestinal epitheliocytes: role of signaling pathways and negative regulators. Front. Immunol. 5:421. doi: 10.3389/fimmu.2014.00421

This article was submitted to Molecular Innate Immunity, a section of the journal Frontiers in Immunology.

Copyright (C) 2014 Villena, Aso and Kitazawa. This is an open-access article distributed under the terms of the Creative Commons Attribution License (CC BY). The use, distribution or reproduction in other forums is permitted, provided the original author(s) or licensor are credited and that the original publication in this journal is cited, in accordance with accepted academic practice. No use, distribution or reproduction is permitted which does not comply with these terms. 